\title{
Progressive encephalomyelitis with rigidity: a case report with magnetic resonance imaging findings
}

\author{
P A MCCOMBE, J B CHALK, J W SEARLE, A E G TANNENBERG, J J SMITH, \\ M P PENDER
}

\author{
From the Departments of Neurology, Neurosurgery and Pathology, Royal Brisbane Hospital and the \\ Department of Medicine, University of Queensland, Royal Brisbane Hospital, Brisbane, Queensland, Australia
}

SUMMARY A 52 year old woman presented with pain in the back and upper limbs and progressive weakness and sensory impairment of the upper and lower limbs. She developed frequent episodes of severe generalised muscle spasms associated with piloerection and hyperventilation. Nerve conduction studies and electromyography were normal. MRI demonstrated a lesion of the cervical spinal cord and lower brainstem. A biopsy of the cord revealed perivascular cuffing with mononuclear cells and inflammatory changes in the parenchyma, with increased numbers of microglia, reactive astrocytes and plasma cells. Following treatment with corticosteroids she showed substantial improvement.

This case report describes a patient with a history and signs suggestive of an intrinsic spinal cord lesion, with unusual clinical and histological features.

\section{Case report}

The patient, a 52 year old female, was admitted after three months of progressive neurological disturbance. She first noticed pain in the interscapular region, then developed pain, paraesthesiae and hyperaesthesia over the shoulders and chest and a feeling of tightness about the chest. She gradually developed numbness of the hands and arms and during the week before admission developed increasing weakness of the upper and lower limbs and urinary retention. There were no involuntary movements at this stage. She had no previous history of neurological symptoms and no significant past medical history. On admission she looked ill, had a heart rate of $84 / \mathrm{min}$ and blood pressure of $160 / 90 \mathrm{~mm} \mathrm{Hg}$. She was breathing with difficulty, but had no other abnormalities on general physical examination. There was no abnormality of the function of higher centres and no neck stiffness. The optic fundi and eye movements were normal. There was reduction in sensation in the territories of the first and second divisions of both trigeminal nerves and the corneal reflexes were depressed. There was weakness of voluntary palatal

Correspondence to: Dr P A McCombe, Department of Medicine, University of Queensland, Clinical Sciences Building, Royal Brisbane Hospital, Brisbane, Queensland, Australia 4029.

Received 3 January 1989 and in revised form 27 April 1989. Accepted 9 June 1989 movement, a poor cough and pooling of secretions in the throat. The upper limbs were flaccid, power was considerably reduced in all muscle groups and the deep tendon reflexes were reduced. In the lower limbs, tone was increased and there was moderate generalised weakness with brisk tendon reflexes and extensor plantar responses. There was severe impairment of sensation to all modalities in the limbs and a zone of hyperaesthesia over the upper chest and back.

A myelogram and CT scan of the spinal cord were normal. An MRI scan showed increased signal intensity throughout the length of the cervical cord and lower brainstem on the T2 weighted scan and reduced signal intensity in this region on the T1 weighted scan (fig 1). A CT brain scan was normal. The CSF contained 404 monouclear white blood cells. The CSF glucose was $3.3 \mathrm{mmol} / \mathrm{l}$ (normal range $2 \cdot 5-5 \cdot 0$ ) and the $\mathrm{CSF}$ protein was $653 \mathrm{mg} / \mathrm{l}$ (normal range $<400$ ). No viruses were cultured from the CSF and cryptococcal antigen was not detected. Serum electrolytes, liver function tests, serum proteins and serum B12 and folate levels were normal. The antinuclear antibody titre was 1:40 and the smooth muscle antibody titre was 1:160. The anticardiolipin antibody level was not elevated. Serum complement and immune complex levels were normal. Antibody to HIV was not present. Nerve conduction studies were normal, and electromyography did not reveal spontaneous muscle activity at rest. Visual evoked responses were normal and an EEG recorded before the start of the muscle spasms was normal.

After admission, the patient suffered increasing respiratory difficulty and needed mechanical ventilation. She was given an intravenous course of $1 \mathrm{gm} /$ day of methylprednisolone on days 3-5 after admission. On the fourth day the cervical cord was explored surgically. 

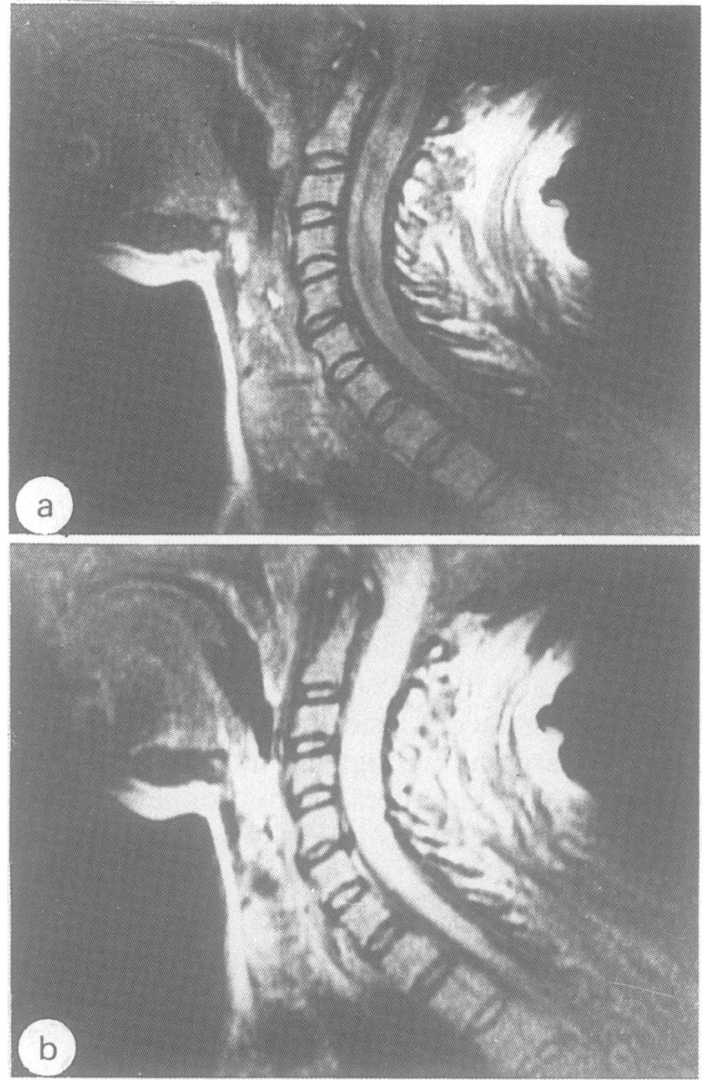

Fig 1 MRI scans of the cervical spinal cord. (A) TI weighted image (TE $30 \mathrm{msec}, T R 600 \mathrm{msec}$ ) showing reduced signal intensity along the cord. (B) T2 weighted image (TE $60 \mathrm{msec}, T R 1500 \mathrm{msec}$ ) showing increased signal intensity of the cervical spinal cord and lower brainstem.

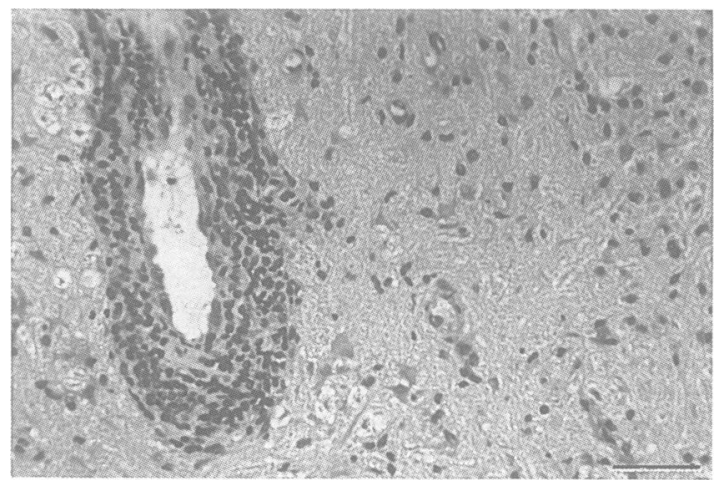

Fig 2 Perivascular lymphocytic cuffing and reactive astrocytes and microglial cells in adjacent neuropil. Paraffin section of biopsy specimen, stained with haematoxylin and eosin. Bar $=50 \mu \mathrm{m}$.
At operation, the surface of the cord looked normal. A midline myelotomy was performed and specimens were taken from the surface and at a depth of $5 \mathrm{~mm}$ into the cord. After surgery, the patient was given dexamethasone $(5 \mathrm{mg}$ every 4 hours on days 6-11) with subsequent gradual withdrawal. She gradually improved and became able to breathe unaided. However, she had lost the increased tone of the lower limbs and was generally flaccid. She developed apnoeic episodes and also episodes of muscle spasms with extension of the upper and lower limbs, associated with piloerection and hyperventilation. These episodes lasted about 60 seconds, occurred many times each day, were distressing for the patient and frequently were precipitated by touch or passive movements of the limbs, or by neurological examination. A further electromyographic examination showed an absence of spontaneous activity when the muscles were relaxed. The patient was given trials of baclofen, sodium valproate and phenytoin. The phenytoin had most effect in reducing the frequency of the spasms. One month after admission, she was given a further course of high dose intravenous methylprednisolone which was associated with further mild improvement in strength. She continued to improve over six months of follow up and was able to walk with support. During a trial of withdrawal of anticonvulsant therapy, after three months of treatment, the muscle spasms returned. A repeat MRI scan (performed five months after the first MRI) was normal.

A histological examination revealed perivascular cuffing with mononuclear cells in both biopsy specimens. Addition- $\square$ ally, the specimen obtained from the deeper levels was 0 hypercellular due to infiltration of the neuropil with $\overrightarrow{\mathbb{D}}$ amoeboid microglia, lipid phagocytes, occasional plasma $\frac{\rho}{\mathrm{D}}$ 윽 cells and plump reactive astrocytes (fig 2). Destruction of $2-$ both myelin and axon cylinders was noted in the deep biopsy.

\section{Discussion}

The clinical features of this case were those of subacute onset of an intrinsic lesion of the cervicaf spinal cord and lower brainstem. The differential diagnosis included a myelitis of subacute onset ${ }^{1}$ or a tumour of the cord. MRI scanning showed an abnormality of the cervical cord extending into the brainstem, with increased signal intensity on the T2 weighted scan and reduced signal intensity on the $T 1$ weighted scan. These appearances may occur with a tumour of the cord ${ }^{2}$ but may also be seen in inflammatory lesions. ${ }^{34}$ The CSF cell count was considerably elevated. This is consistent with an inflammatory process, but does not usually occur with a spinal cord tumour. ${ }^{5}$ The biopsy material showed a typical inflammatory process with perivascular cuffing with mononuclear cells and increased numbers of reactive astrocytes, microglia and plasma cells in the parenchyma. The subsequent clinical improvement, which may have been related to corticosteroid treatment was consistent with an inflammatory process. The aetiology of the inflammatory process is not clear. There was no evidence of an infection and it is possible that the inflammation was due to an autoimmune process. The patient had a weakly positive antinuclear 
factor which may be associated with systemic lupus erythematosus, a cause of myelopathy, ${ }^{367}$ but there were no other features of a connective tissue disorder.

A prominent feature of this case was the occurrence of frequent episodes of involuntary stiffenings of all limbs, associated with piloerection and hyperventilation. These were reminiscent of the periodic increases in muscle tone which may occur in unconscious patients with brainstem damage ${ }^{8}$ and also had some similarities to tonic seizures. ${ }^{9}$ The spasms were reduced in frequency and severity by treatment with phenytoin. The mechanism of the spasms is unknown but because of the simultaneous stiffenings of all limbs into a decerebrate posture, and the association with piloerection and hyperventilation, it is possible that the brainstem may have been the site of the disturbance.

There are previous reports of muscle spasms in patients with spinal cord inflammation. ${ }^{10-13}$ All cases were characterised by the subacute onset of frequent, severe, painful muscle spasms precipitated by sensory stimulation and by neurological examination and all had a fatal outcome. In some patients there was mild muscle stiffness in the periods between the painful spasms. Some patients assumed a position of flexion of the upper limbs, which was not the case in our patient who assumed a position of extension. Electromyography in one patient ${ }^{10}$ showed continuously active normal motor units during the spasm, which disappeared when the patient became quiet, while in another ${ }^{11}$ there was spontaneous motor activity even at rest. In our patient there was no EMG activity at rest. In all patients histological examination showed perivascular lymphocytic cuffing and gliosis, similar to the findings in our patient. Progressive encephalomyelitis with rigidity can be distinguished from the stiff-man syndrome ${ }^{14}$ as it is associated with an underlying neuropathological lesion. However, recent reports have suggested that the stiff-man syndrome may be an autoimmune disorder. ${ }^{15-16}$ Other reports ${ }^{17} 18$ described patients with myoclonus and rigidity, and in one case, ${ }^{18}$ a paraneoplastic syndrome. Thus progressive encephalomyelitis with rigidity is a syndrome with known clinical, pathological and MRI appearances. Previous reported cases have been fatal but the outcome of the present case suggests that with supportive care and high dose corticosteroid treatment patients may survive and eventually show substantial improvement.

\section{References}

1 Ropper AH, Poskanzer DC. The prognosis of acute and subacute transverse myelopathy based on early signs and symptoms. Ann Neurol 1978;4:5i-9.

2 Scotti G, Scialfa G, Colombo N, Landoni L. Magnetic resonance diagnosis of intramedullary tumors of the spinal cord. Netiroradiology 1987;29:130-5.

3 Kenik JG, Krohn K, Kelly RB, Bierman M, Hammeke MD, Hurley JA. Transverse myelitis and optic neuritis in systemic lupus erythematosus: a case report with magnetic resonance imaging findings. Arthritis Rheum 1987;8:947-50.

4 Hosada K, Tamaki N, Masamura M, Matsumoto S. Magnetic resonance images of brain-stem encephalitis. Case Report. J Neurosurg 1987;66:283-5.

5 Findlay GFG. Compressive and vascular disorders of the spinal cord. In: Miller JD, ed. Northfield's Surgery of the Central Nervous System. Oxford: Blackwell, 1987: 707-57.

6 Al-Husaini A, Jamal GA. Myelopathy as the main presenting feature of systemic lupus erythematosus. Eur Neurol 1985;24:94-106.

7 Hardie RJ, Isenberg DA. Tetraplegia as a presenting feature of systemic lupus erythematosus complicated by pulmonary hypertension. Ann Rheum Dis 1985;44: 491-3.

8 Miller JD. Head injury. In Miller JD, ed. Northfield's Surgery of the Central Nervous System. Oxford: Blackwell, 1987:795-870.

9 Matthews WB. Tonic seizures in disseminated sclerosis. Brain 1958;81:193-206.

10 Kasperek S, Zebrowski S. Stiff-man syndrome and encephalomyelitis. Report of a case. Arch Neurol 1971;24:22-30.

11 Lhermitte F, Chain F, Escourolle R, Chedru F, Guilleminault C, Francoual $M$. Un nouveau cas de contracture tétaniforme distinct du stiff-man syndrome. Etude pharmacologique et neuropathologique d'un cas d'encéphalomyélite à prédominance médullaire. Revue Neurologique 1973;128:3-21.

12 Whiteley AM, Swash M, Urich H. Progressive encephalomyelitis with rigidity. Its relation to "subacute myoclonic spinal neuronitis" and to the "stiff-man syndrome". Brain 1976;99:27-42.

13 Howell DA, Lees AJ, Toghill PJ. Spinal internuncial neurones in progressive encephalomyelitis with rigidity. J Neurol Neurosurg Psychiatry 1979;42:773-85.

14 Moersch FP, Woltman HW. Progressive fluctuating muscular rigidity and spasm ('stiff-man' syndrome): report of a case and some observations in 13 other cases. Proc Staff Meetings Mayo Clinic. 1956;31:421-7.

15 Layzer RB. Stiff-man syndrome-an auto-immune disease. New Engl J Med 1988;318:1060-2.

16 Solimena M, Folli F, Denis-Donini S, Comi GC, Pozza G, De Camilli P, Vicari AM. Autoantibodies to glutamic acid decarboxylase in a patient wth stiff-man syndrome, epilepsy, and Type I diabetes mellitus. New Engl J Med 1988;318:1012-20.

17 Campbell AMG, Garland H. Subacute myoclonic spinal neuronitis. J Neurol Neurosurg Psychiatry 1956;19: 268-74.

18 Roobol TH, Kazzaz BA, Vecht CHJ. Segmental rigidity and spinal myoclonus as a paraneoplastic syndrome. $J$ Neurol Neurosurg Psychiatry 1987;50:628-31. 